\title{
Fuel flow control through a fuzzy servomechanism: a comparative analysis
}

\author{
Víctor M. Castaño ${ }^{1 *}$, Domingo Rangel-Miranda ${ }^{1}$, Daniel Alaniz-Lumbreras ${ }^{2}$, Ernesto Olvera-Gonzálezb ${ }^{2}$ \\ ${ }^{1}$ Centro de Física Aplicada y Tecnología Avanzada, Universidad Nacional Autónoma de México, \\ Campus Juriquilla, Querétaro, México \\ ${ }^{2}$ Doctorado en Ciencias de la Ingeniería, Unidad Académica de Ingeniería Eléctrica, \\ Universidad Autónoma de Zacatecas, Zacatecas, México \\ *Corresponding authorE-mail: meneses@unam.mx
}

Copyright (C) 2014 Víctor M. Castaño et al. This is an open access article distributed under the Creative Commons Attribution License, which permits unrestricted use, distribution, and reproduction in any medium, provided the original work is properly cited.

\begin{abstract}
The evaluation of a fuzzy expert positioning servomechanism that was applied for controlling fuel flow in an experimental process is presented. Since the conventional control techniques are not sufficient to implement nonlinear control systems, a successful control technique based on human experience was used. A comparative study was made by both Fuzzy Logic Control (FLC) and Proportional Integral Derivative (PID) control algorithms. Fuzzy Logic and PID controllers were designed in Lab view program. The real-time position control which consists of a DC motor into a servomechanism was implemented by using a digital acquisition device (DAQ). The experimental results show that step response and semicircular trajectory on the position servomechanism for controlling fuel using FLC, had a better performance than those derived from PID control scheme.
\end{abstract}

Keywords: Expert System, Fuel Control Schemes, Fuel Flow Supply, Fuzzy Logic Control, and Rule-Based Control.

\section{Introduction}

One of the most important challenges in environmental sciences is the rational use of fuels [1]. In particular, the use of biofuels, such as ethanol, can represent a viable alternative source of energy for both industrial and domestic applications [2]. Whatever the source, fuel supply requires of adaptable control techniques to supplement the conventional techniques for increasing the performance. These techniques must be able to reproduce effectively the experience of the human operator, difficult task especially since these systems present a nonlinear behavior [3].

Fuzzy logic is a soft computing technique, which mimics the ability of the human mind to learn and make rational decisions in an uncertain and imprecise environment. Fuzzy Logic, the logic of approximate reasoning, is a technology that enables users to describe a desired system behavior using every day spoken language [4]. A Fuzzy Logic Control (FLC), Takagi-Sugeno type consists of a set of linguistic statements which are composed of two inputs, the error and the rate error conditions to carry out control actions by "if -then" rules [5].

Several applications of fuzzy control have been implemented successfully in order to control physical parameters, such as amount of fuel required for small engines by an optimized fuel injection system [6], in which the fuzzy algorithm uses the engine speed and manifold air pressure as input values. The performance of the engine running with the Fuzzy Control System (FCS) was experimentally compared to that of the engine running using the conventional mechanical fuel regulation and delivery system. The fuzzy control had better performance than the conventional system.

An alternative method applied to a gas engine driven heat pump was proposed for a system that does not take into account the whole model [7]. The cascade fuzzy control is the control strategy which was compared to a cascade PI (proportional and integral) control strategy. As a consequence, the reduction and saving energy were reported as main advantages.

The fuzzy adaptable control has been also employed for position control in an ultrasonic motor [8]. The dynamic characteristics of an ultrasonic motor are difficult to obtain and the motor parameters are time-dependent, so that a fuzzy adaptive model-following position controller was implemented for reducing the influence of parameters 
variations. However, the fuzzy adaptive control was not optimized to have better performance. Also, an expert system has been developed for defining possible fuel system faults [9], in which neither characteristics for fuel source nor the system behavior at different environmental conditions, such as fuel temperature, were taken into account. On the order hand, the design, implementation and evaluation of two types of fuzzy controllers [10] were proven superior in comparison to the conventional controller by a PID control strategy, in a jet engine test bench having all environmental parameters well controlled.

Here, an alternative fuzzy control strategy to supply fuel to an experimental process by using an optimized expert system is presented. The fuzzy scheme rule-based is aimed to increase the accuracy and performance of a control servomechanism of environmental parameters such as fuel temperature, density and differential pressure in an ethanol supply control system.

\section{The expert positioning system}

\subsection{Fuel supply control servomechanism}

The control scheme strategy of a fuzzy expert position servomechanism was implemented in Lab View platform for testing the transient and steady state responses on the system in real time. The control parameters can be changed during the running test in a PC.

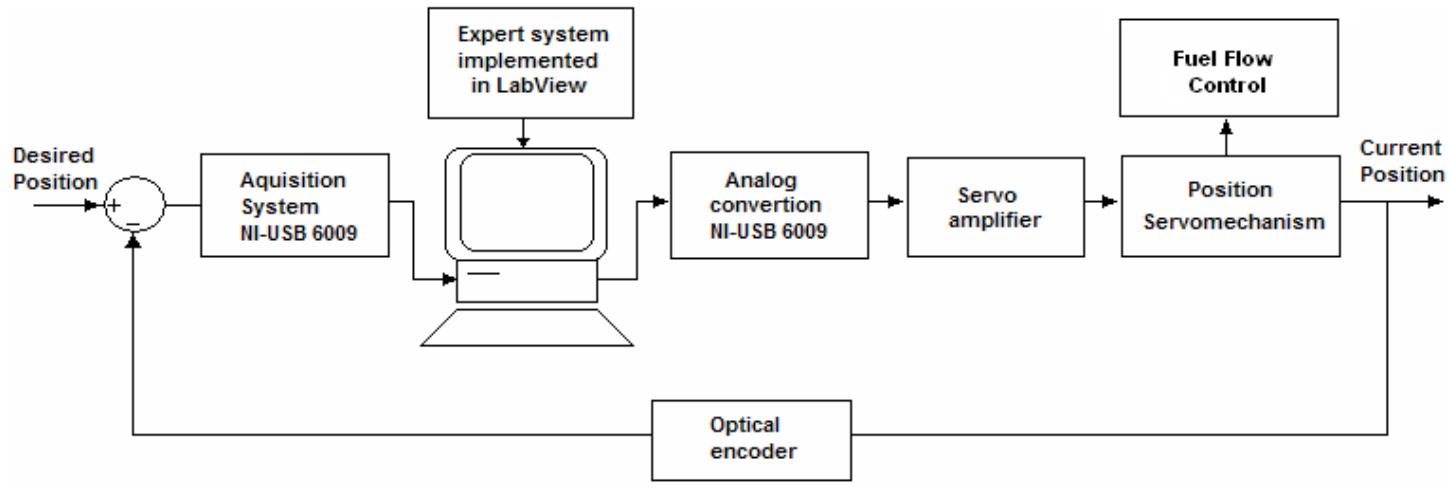

Fig. 1: Block Diagram of the Fuzzy Expert Position Servomechanism.

Fig. 1 shows the closed loop position servomechanism in which a DAQ NI-USB 6009 was used to convert the external data from an optical encoder, which gives information about the current position. The position servomechanism consists of a Pittman DC motor Model GM9x34 which is coupled into a flow regulator system capable of supplying the quantity of fuel to the process. The implemented algorithms in Lab View were Proportional Integral Derivative (PID) and Fuzzy Logic Control (FLC).

Dynamically, a DC motor can be characterized as a second order system. The rotor current is generated according to the rotor (armature) voltage, the induced reverse voltage, the ohmic internal resistance, and the inductance of the rotor. The motor torque is proportional to the rotor current. The velocity of the motor is derived by integrating the motor angular acceleration. Typically, the rotor current is controlled by a PI controller [11], but the fuel supply control system have nonlinearities due to the variable load in DC motor, such as torque limitation, intermittent flow and dry friction. The block diagram of the motor with the current loop is shown in Fig. 2.

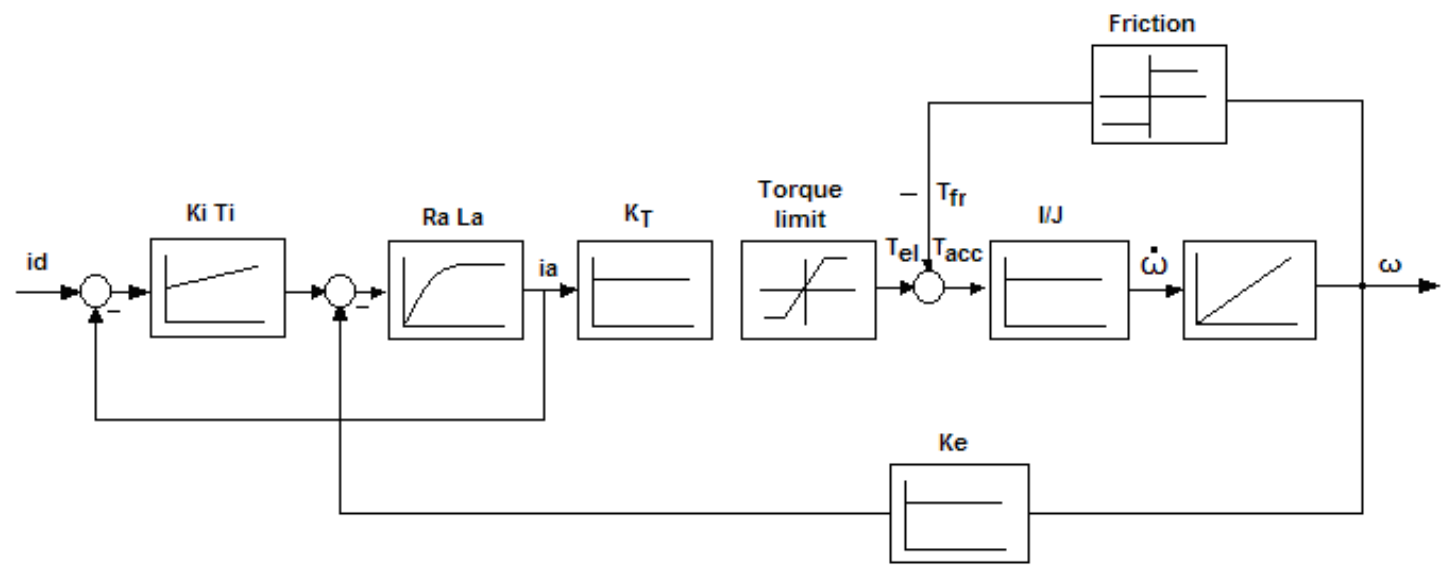

Fig. 2: DC Motor Nonlinear Parameters into the Fuel Flow Regulator System. 
The control aim for the fuel flow control by mean a DC motor is:

- Overshoot $0.5 \%$ or less

- No steady state error

- Minimum settling time

- $\quad$ Minimum rising time

\subsection{Design of the fuzzy expert system}

An expert system is based on operator experience that has knowledge about the system. Many software computing techniques such as fuzzy logic, neural networks and genetic algorithms have been developed for attempting to mimic the human reasoning to solve engineering and industrial problems successfully [12], [13], [14]. The FLC consists of mainly three basic components, namely the Fuzzification, Fuzzy Engine (rule-base) and Defuzzification. The Fuzzification transforms the analog input signal into linguistic fuzzy variable between 0 and 1 so-called membership function $(\mu)$. The fuzzy engine handles the rule inference where the variables are articulated in "if-then" rules. The Defuzzification transforms the fuzzy control actions to continuous signal that can be applied to the system.

Fuzzy logic controller according to the Takagi-Sugeno model has two inputs and one output [15]. The inputs are the position error (e) and the change of the position error $(\Delta \mathrm{e})$ in a sampling time interval, and output is the control signal $(\mathrm{u})$. At the sampling point $\mathrm{k}$, the position error $(\mathrm{e})$ and change of the error $(\Delta \mathrm{e})$ are calculated as:

$$
\begin{aligned}
& e(k)=\theta_{d}(k)-\theta_{a}(k) \\
& \dot{e}(k)=e(k)-e(k-1)
\end{aligned}
$$

Where:

$\theta_{\mathrm{d}}(\mathrm{k})$ is the desired angular position and $\theta_{\mathrm{a}}(\mathrm{k})$ is the current angular position. To define membership functions for (e), $(\Delta \mathrm{e})$ and $(\mathrm{u})$, the universe of discourse was divided in seven domains which are NB (negative big), NM (negative medium), NS (negative small), Z (zero), PS (positive small), PM (positive medium) and PB (positive big) as shown in Fig. 3, 4 and 5 .

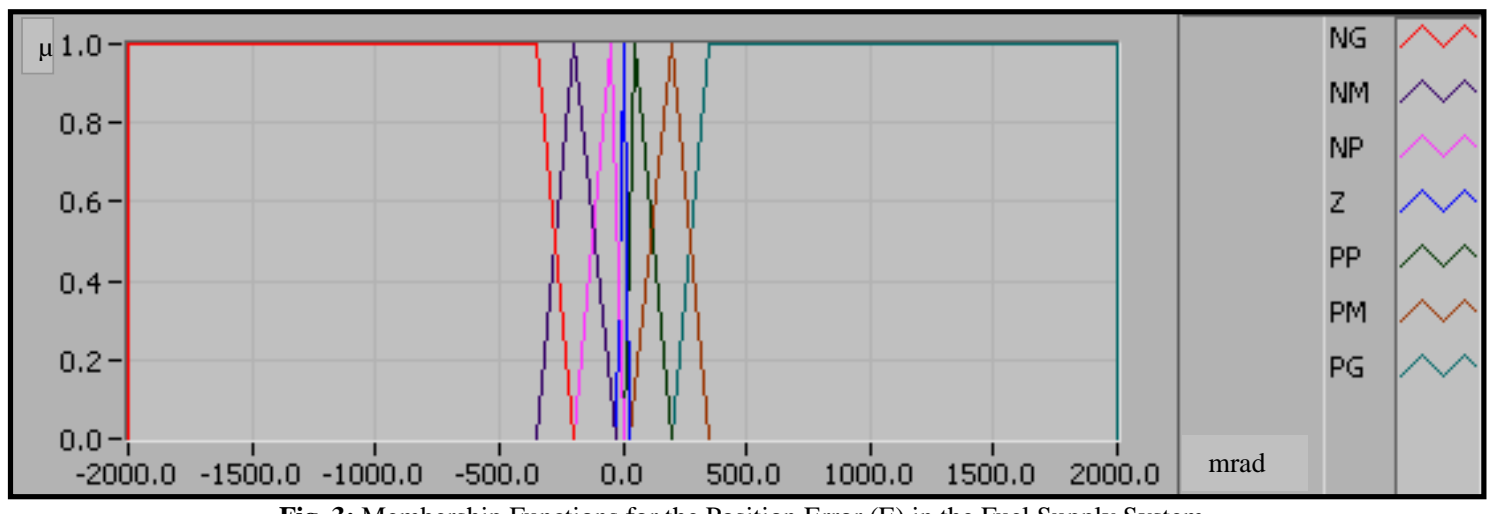

Fig. 3: Membership Functions for the Position Error (E) in the Fuel Supply System.

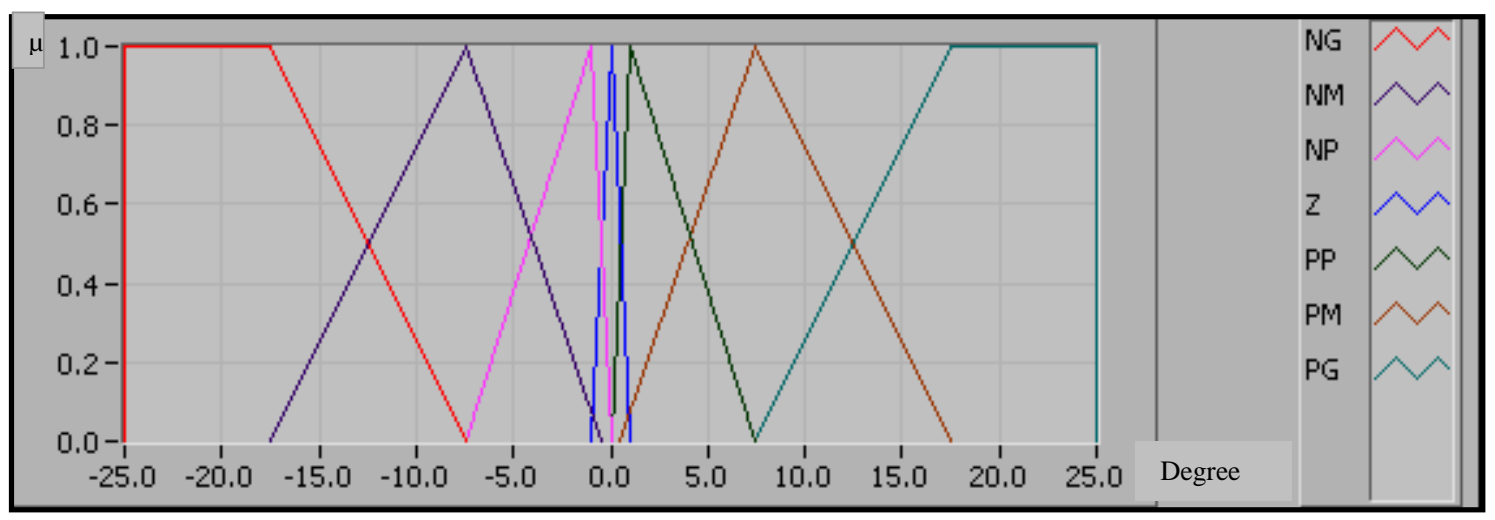

Fig. 4: Membership Functions for the Change of the Position Error $(\Delta \mathrm{E})$ in the Fuel Supply System. 


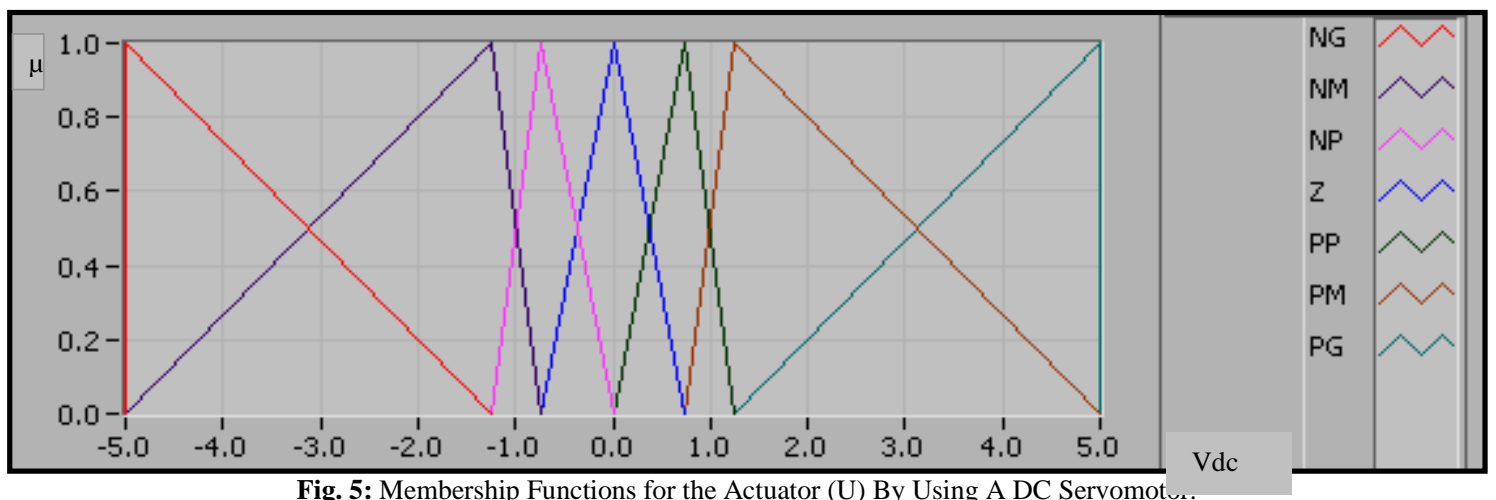

The Fuzzy Associated Matrix (FAM) was built in several load conditions by using linguistic information of the human operator. Both the fuzzyfication and inference system were tuned experimentally. This framework could be tedious and time-consuming because intrinsic nonlinearities in the position servomechanism are difficult to model and implement on the physical system in real-time [16].

The error position indicates the magnitude of the difference between desired position and current position in each sampling time. The rate of error indicates if the error is increasing positively or negatively as the system reaches the desired position. The fuzzy sets and the membership functions were built by using the associated information based on the operator experience during the fuel supply to the experimental process control.

The information in linguistic form is converted to a membership function between 0 and 1 , according to the weight of each rule in the output system. The algorithm used for inference is MAX-MIN method, equivalent to AND function in binary logic where the maximum value of the minimum element is the most representative element in the output function [17].

Counterclockwise and clockwise rotations are defined as positive and negative, respectively. A corresponding output is given for the error and error change detected in each sampling interval. For example, if the error is positively large and the error change is positively small, then a large positive drive is used. Table 1 shows the fuzzy associated matrix for the error and the change of error of the fuel flow control system.

Table 1: Fuzzy Associated Matrix for the Error and the Change of Error of the Fuel Flow System.

\begin{tabular}{cllccccc}
\hline$e / e$ & NG & NM & NP & Z & PP & PM & PG \\
\hline NG & NG & NG & NB & NG & NP & PP & PG \\
NM & NG & NG & NM & NM & Z & PP & PG \\
NP & NG & NG & NS & NS & Z & PM & PG \\
Z & NG & NG & NS & Z & PP & PG & PG \\
PP & NG & NM & Z & PP & PP & PG & PG \\
PM & NG & NP & Z & PM & PM & PG & PG \\
PG & NG & NP & PP & PG & PG & PG & PG \\
\hline
\end{tabular}

\section{Experimental results}

The control signal obtained from DAQ device is amplified by a power amplifier and feed to the motor voltage with \pm 12 VDC. To make a good comparison between performances of PID and FLC, step input and semicircular trajectory were applied to the system at the same electrical and mechanical conditions. The responses at two different conditions were implemented and analyzed for supplying fuel in real-time. Since these inputs are two of the most important functions in supplying fuel systems they were evaluated according to the rising time, overshoot and settling time. As is shown in Fig. 6, the position motor was settled at $120 \mathrm{~ms}$ when PID controller was used.

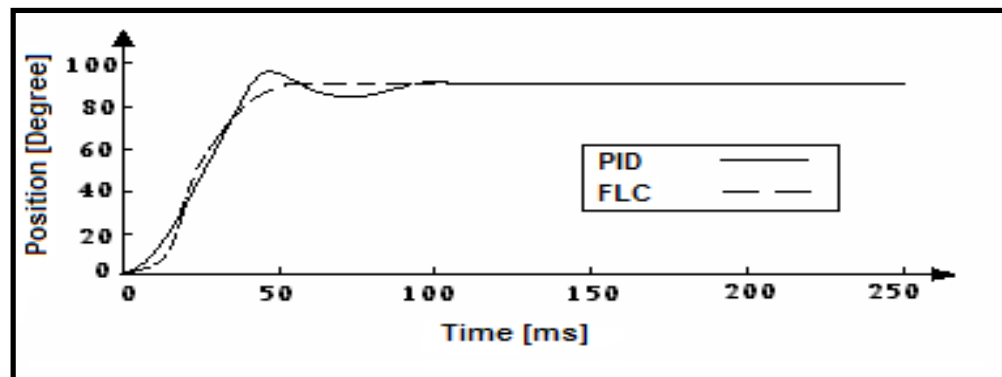

Fig. 6: Response of the Fuzzy Expert Position Servomechanism to Step Input. 
This performance is out of the aimed control level. However, if the coefficients of PID controller are tuned experimentally, PID can achieve the control aim. After this tune the overshoot decreases and the settling time increases proportionally. On the other hand, the desired control aim was achieved with $0.4 \%$ overshoot and $80 \mathrm{~ms}$ settling time by using FLC. Integral Absolute Error (IAE) and Integral Time Absolute Error (ITAE) values were calculated from 38 ms to $250 \mathrm{~ms}$. In order to compare both control schemes the IAE and ITAE are shown in Table 2. Considering only these parameters, FLC gives better performance than PID control. Also, overshoot and settling time values are shown to establish that only FLC achieved the control aim for the same rise time than PID control.

Table 2: Control Positioning Parameters for Performing Evaluation of the Expert Servomechanism.

\begin{tabular}{lll}
\hline \multirow{2}{*}{ Step input [parameter] } & \multicolumn{2}{c}{ Type of control scheme } \\
\cline { 2 - 3 } & Fuzzy Logic & PID \\
\hline Rising time $\mathrm{t}_{\mathrm{r}}[\mathrm{ms}]$ & 39 & 38 \\
Overshoot [\%] & 0.4 & 4 \\
Settling t $[\mathrm{ms}]$ & 80 & 120 \\
Integral absolute error (IAE) & $34.2(38-250 \mathrm{~ms})$ & $57.78(38-250 \mathrm{~ms})$ \\
Integral time absolute error (ITAE) & $772.2(38-250 \mathrm{~ms})$ & $1851.8(38-250 \mathrm{~ms})$ \\
Semicircular trajectory & & \\
[parameter] & Fuzzy Logic & PID \\
Integral absolute error (IAE) & 719.7 & 554.83 \\
Integral time absolute error (ITAE) & 39919 & 25929 \\
\hline
\end{tabular}

A semicircular trajectory (from $0^{\circ}$ to $180^{\circ}$ ) was implemented in the positioning servomechanism. Since that trajectory is one of the most common position trajectories in fuel supply control systems, it was chosen to regulate fuel flow. Semicircular response is shown in Fig. 7, the system with PID control was not able to follow the semicircular trajectory and it oscillated.

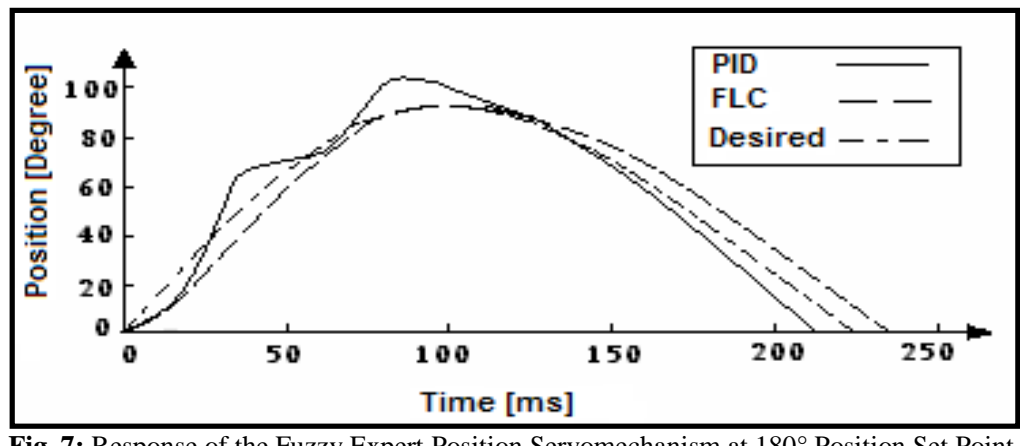

The reason of these deviations could be due to the integral effect in PID controller. From a different point of view, the reference was followed linearly, decreasing the positive error at the first $90^{\circ}$, and with linearly increasing the negative error at the second $90^{\circ}$ of the semicircular input when FLC is used. For these responses, IAE and ITAE values are given in Table 2. These values show that the PID controller is better than FLC.

\section{Conclusions}

The performance of a fuzzy expert servomechanism for a fuel supply system was evaluated. Since both industrial and domestic fuel supply systems, such as ethanol engines are becoming more and more common, the approach is capable to convert the environmental characteristics, such as fuel temperature, density and differential pressure into linguistic rules. A positioning servomechanism was implemented in Lab View for tuning control parameters in real-time by both PID and FLC schemes by using a knowledge-base according to expert human operator. Also, to tune FLC, experimental data and repetitive experiments are needed. The development and implementation of a fuzzy expert for position control coupled to a DC servomotor were successfully implemented using Lab View and a DAQ device in closed loop. The system was proved with step and semicircular trajectories. Experimental results based on IAE and ITAE suggest that FLC responds with less overshot and minimum settling time for step response on the fuel flow control servomechanism.

\section{Acknowledgments}

The authors want to thank Universidad Autónoma de Querétaro for their facilities. One of us (DR) was supported by a CONACYT (118739) fellowship. 


\section{References}

[1] Ajit, K.B., Pramod, S.M., Chandra, P.G., A rational approach in predicting fuel sprays shape. Fuel 65 (1986) 1017-1018. http://dx.doi.org/10.1016/0016-2361 (86)90215-2.

[2] Lapuerta, M., Armas, O., and Herreros, J.M., Emissions from a diesel-bioethanol blend in an automotive diesel engine. Fuel 87 (2008) 25-31. http://dx.doi.org/10.1016/j.fuel.2007.04.007.

[3] Anurag, G., Gopal, A., and Ramesh, C.J., Application of fuzzy logic to estimate flow of methane for energy generation at a sanitary landfill. J. Energy Engineering 133 (2007) 212-223. http://dx.doi.org/10.1061/ (ASCE) 0733-9402(2007)133:4(212).

[4] Zadeh, L.A., Fuzzy sets. Information and Control 8 (1965) 338-353. http://dx.doi.org/10.1016/S0019-9958 (65)90241-X.

[5] Takagi, T., and Sugeno, M., Fuzzy identification of systems and its application to modeling and control. IEEE Trans. Syst., Man, Cyber. 15 (1985) 116-132. http://dx.doi.org/10.1109/TSMC.1985.6313399.

[6] Lee, S.H., Howlett, R.J., and Walters S.D., Small engine control by fuzzy logic. Journal of Intelligent and Fuzzy Systems 15 (2004) $207-217$.

[7] Shuze, L., Rongrong, Z., Dexu, L., and Huang, Z., Cascade fuzzy control for gas engine driven heat pump. Energy, Convertion and Management 46 (2005) 1757-1766. http://dx.doi.org/10.1016/j.enconman.2004.09.003.

[8] Sánchez, E., and Flores, V., Real time underactuated robot swing-up via fuzzy PI+PD control. Journal of Intelligent and Fuzzy Systems 17 (2006) 1-13.

[9] Kilagiz, Y., Bran, H., Yildiz, Z., and Cetín, M., A fuzzy diagnosis and advice system for optimization of emissions and fuel consumption. Expert Systems with Applications 28 (2005) 305-311. http://dx.doi.org/10.1016/j.eswa.2004.10.016.

[10] Zilouchian, A., Juliano, M., Healy, T., and Davis J., Design of a fuzzy logic controller for a jet engine fuel system. Control Engineering Practice 8 (2000) 873-883. http://dx.doi.org/10.1016/S0967-0661 (00)00019-8.

[11] Tanie, K., Yokoi, K., and Kaneko, M., A position based torque control method for a dc motor with reduction gears. Journal of Robotics and Mechatronics 1 (1989), 29-33.

[12] Andrés-Toro, B., Gigón-Sierra, J.M., López-Orozco, J.A., and Fernández-Conde, C., Using genetic algorithms for dynamic optimization: an industrial fermentation case. In: Proc. of the 36th IEEE Conference on Decision and Control, San Diego California, (1997) 828-829. http://dx.doi.org/10.1109/CDC.1997.650742.

[13] Ouladsine, M., Bloch, G., and Dovifaaz, X., Neural modeling and control of a diesel engine with pollution constraints. Journal of Intelligent and Robotic Systems 41 (2004) 157-171. http://dx.doi.org/10.1007/s10846-005-3806-y.

[14] Zhang, J.H., Zhang, H., Su, D.S., Qin, Y., Huo, M.Y., Zhang, Q.H., and Wang, L., Adaptive fuzzy control system of a servomechanism for electro-discharge machining combined with ultrasonic vibration. Journal of Materials Processing Technology 129 (2002) 45-49. http://dx.doi.org/10.1016/S0924-0136 (02)00573-3.

[15] Johansen, T.A., Shorten, R., and Murray-Smith, R., on the interpretation and identification of dynamic Takagi-Sugeno fuzzy models. IEEE Transactions on Fuzzy Systems 8 (2000) 297-313. http://dx.doi.org/10.1109/91.855918.

[16] Wang, L., Automatic design of fuzzy controllers. Automatica 35 (1999) 1471-1475. http://dx.doi.org/10.1016/S0005-1098 (99)00044-8.

[17] Tanaka, K., and Wang, H.O., Fuzzy control systems design and analysis: a linear matrix inequality approach, Wiley Inter-Science, New York, (2001) 5-48. http://dx.doi.org/10.1002/0471224596.ch2. 